\title{
dtControl 2.0: Explainable Strategy Representation via Decision Tree Learning Steered by Experts *
}

\author{
Pranav Ashok ${ }^{1}$ ID Mathias Jackermeier ${ }^{1}$, Jan Křetínský ${ }^{1}$,

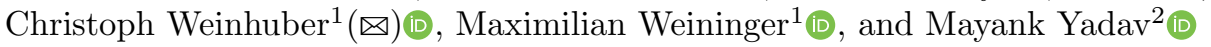 \\ 1 Technical University of Munich, Munich, Germany \\ firstname.lastname@tum.de \\ 2 Department of Computer Science and Engineering, I.I.T. Delhi, \\ New Delhi, India \\ cs1180356@iitd.ac.in
}

\begin{abstract}
Recent advances have shown how decision trees are apt data structures for concisely representing strategies (or controllers) satisfying various objectives. Moreover, they also make the strategy more explainable. The recent tool dtControl had provided pipelines with tools supporting strategy synthesis for hybrid systems, such as SCOTS and Uppaal Stratego. We present dtControl 2.0, a new version with several fundamentally novel features. Most importantly, the user can now provide domain knowledge to be exploited in the decision tree learning process and can also interactively steer the process based on the dynamically provided information. To this end, we also provide a graphical user interface. It allows for inspection and re-computation of parts of the result, suggesting as well as receiving advice on predicates, and visual simulation of the decision-making process. Besides, we interface model checkers of probabilistic systems, namely STORM and PRISM and provide dedicated support for categorical enumeration-type state variables. Consequently, the controllers are more explainable and smaller.
\end{abstract}

Keywords: Strategy representation - Controller representation · Decision Tree · Explainable Learning $\cdot$ Hybrid systems $\cdot$ Probabilistic Model Checking · Markov Decision Process

\section{Introduction}

A controller (also known as strategy, policy or scheduler) of a system assigns to each state of the system a set of actions that should be taken in order to achieve a certain goal. For example, one may want to satisfy a given specification of a robot's

* This work has been partially supported by the German Research Foundation (DFG) project No. 383882557 SUV (KR 4890/2-1), No. 427755713 GOPro (KR 4890/3-1) and the TUM International Graduate School of Science and Engineering (IGSSE) grant 10.06 PARSEC. We thank Tim Quatman for implementing JSON-export of strategies in STORM and Pushpak Jagtap for his support with the SCOTS models.

(C) The Author(s) 2021

J. F. Groote and K. G. Larsen (Eds.): TACAS 2021, LNCS 12652, pp. 326-345, 2021.

https://doi.org/10.1007/978-3-030-72013-1_17 
behaviour or exhibit a concurrency bug appearing only in some interleaving. It is desirable that the controllers possess several additional properties, besides achieving the goal, in order to be usable in practice. Firstly, controllers should be explainable. Only then can they be understood, trusted and implemented by the engineers, certified by the authorities, or used in the debugging process 11]. Secondly, they should be small in size and efficient to run. Only then they can be deployed on embedded devices with limited memory of a few kilobytes, while the automatically synthesized ones are orders of magnitude larger [49]. Thirdly, whenever the primary goal, e.g. functional correctness, is accompanied by a secondary criterion, e.g. energy efficiency, they should be performant with respect to this criterion.

Automatic controller synthesis is able to provide controllers for a given goal in various domains, such as probabilistic systems 32,17 , hybrid systems [45. $16,30,19]$ or reactive systems [35]. In some cases, even the performance can be reflected [16. However, despite recent interest in explainability in connection to AI-based controllers 2 and despite typically small memories of embedded devices, automatic techniques for controller synthesis mostly fall short of producing small explainable results. A typical outcome is a controller in the form of a look-up table, listing the actions for each possible state, or a binary decision diagram (BDD) [14 representation thereof. While the latter reduces the size to some extent, none of the two representations is explainable: the former due to its size, the latter due to the bit-level representation with all high-level structure lost. Instead, learning representations in the form of decision trees (DT) [38 has been recently explored to this end $[7,3$. DTs turn out to be usually smaller than BDD but do not drown to the bit level and are generally well known for their interpretability and explainability due to their simple structure. However, despite showing significant potential, the state-of-the-art tool dtControl [4] uses predicates without natural interpretation, and moreover, the best size reductions are achieved using determinization, i.e. making the controller less permissive, which negatively affects performance 7 .

Example 1 (Motivating example). Consider the cruise control model of [34], where we want to control the speed of our car so that it never crashes into the car in front while, as a secondary performance objective, keeping the distance between the two cars small.

A safe controller for the this model as returned by Uppaal Stratego, is a lookup table of size $418 \mathrm{MB}$ with 300,000 lines. The respective BDD has 1,448 nodes with all information bit-blasted. Using adaptations of standard DT-construction algorithms, as implemented in dtControl, we can get a DT with 987 nodes, which is still too large to be explained. Using determinization techniques, the controller can be compressed to 3 nodes! However, then the DT allows only to decelerate until the minimum velocity. This is safe, as we cannot crash into the car in front, but it does not even attempt at getting close to the front car, and thus has a very bad performance.

One can find a strategy with optimal performance, retaining the maximal permissiveness, not determinizing at all, which can be represented by a DT with 11 
nodes. A picture of this DT as well as reasoning how to derive the predicates from the kinematic equations is in the extended version of this paper [5 Appendix A].

However, exactly because the predicates are based on the domain knowledge, namely the kinematic equations, they take the form of algebraic predicates and not simply linear predicates, which are the only ones in dtControl and commonly in the machine-learning literature on DTs.

This motivating example shows that using domain knowledge and algebraic predicates, available now in dtControl 2.0, one can get smaller representation than when using existing heuristics. Further, it improves the performance of the DT, and it is easily explainable, as it is based on domain knowledge. In fact, the discussed controller is so explainable that it allowed us to find a bug in the original model. In general, using dtControl 2.0 a domain expert can try to compress the controller, thus gain more insight and validate that it is correct. Another example of this has been reported from the use of dtControl in the manufacturing domain 31.

While automatic synthesis of good predicates from the domain knowledge may seem as distant as automatic synthesis of program invariants or automatic theorem provers, we adopt the philosophy of those domains and offer semi-automatic techniques.

Additionally, if not performance but only safety of a controller is relevant, we can still benefit from determinization without drawbacks. To this end, we also provide a new determinization procedure that generalizes the extremely successful MaxFreq technique of [4] and is as good or better on all our examples.

To incorporate the changes just discussed, namely algebraic predicates, semiautomatic approach, and better determinization, we have also reworked the tool and its interfaces. To begin with, the software architecture of dtControl 2.0 is now very modular and allows for easy further modifications, as well as adding support for new synthesis tools. In fact, we have already added parsers for the tools STORM [17] and PRISM [32], and thus we support probabilistic models as well. Since these models also contain categorical (or enumerationtype) variables, e.g. protocol states, we have also added support for categorical predicates. Furthermore, we added a graphical user interface that not only is easier to use than the command-line interface, but also allows to inspect the DT, modify and retrain parts of it, and simulate runs of the model under its control, further increasing the possibilities to explain the DT and validate the controller.

Summing up, the main improvements of dtControl 2.0 over the previous version [4] are the following:

- Support of algebraic predicates and categorical predicates

- Semi-automatic interface and GUI with several interactive modes

- New determinization procedure

- Interfaces for model checkers PRISM and Storm and experimental evidence of improvements on probabilistic models compared to BDD

The paper is structured as follows. After recalling necessary background in Section 2, we give an overview of the improvements over the previous version of 
the tool from the global perspective in Section 3. We detail on the algorithmic contribution in Sections 4 (predicate domains), 5 (predicate selection) and 6 (determinization). Section 7 provides experimental evaluation and Section 8 concludes.

Related work. DTs have been suggested for representing controllers of and counterexamples in probabilistic systems in [11], however, the authors only discuss approximate representations. The ideas have been extended to other setting, such as reactive synthesis [12] and hybrid systems 7]. More general linear predicates have been considered in leaves of the trees in $|3|$. dtControl 2.0 contains the DT induction algorithms from $[7,3]$. The differences to the previous version of the tool dtControl [4] are summarized above and schematically depicted in Figure 2,

Besides, DTs have been used to represent and learn strategies for safety objectives in 40 and to learn program invariants in [21]. Further, DTs were used for representing the strategies during the model checking process, namely in strategy iteration [10] or in simulation-based algorithms 42. Representing controllers exactly using a structure similar to DT (mistakenly claimed to be an algebraic decision diagram) was first suggested by [22], however, no automatic construction algorithm was provided.

The idea of non-linear predicates has been explored in [28]. In that work, however, it is not based on domain knowledge, but rather on projecting the state-space to higher dimensions.

BDDs [14 have been commonly used to represent strategies in planning [15], symbolic model checking 32 as well as to represent hybrid system controllers [45, 30]. While BDD [14 operate only on Boolean variables, they have the advantage of being diagrams and not trees. Moreover, they correspond to Boolean functions that can be implemented on hardware easily. [18] proposes an automatic compression technique for numerical controllers using BDDs. Similar to our work, 49 considers the problem of obtaining concise BDD representation of controllers and presents a technique to obtain smaller BDDs via determinization. However, BDDs are difficult to explain due to variables being bit-blasted and their size is very sensitive to the chosen variable ordering. An extension of BDDs, algebraic or multi-terminal decision diagrams (ADD/MTBDD) 8, 20, have been used in reinforcement learning for strategy synthesis [26, 47]. ADDs extend BDDs with the possibility to have multiple values in the terminal nodes, but the predicates still work only on boolean variables, retaining the disadvantages of BDDs.

\section{Decision tree learning for controller representation}

In this section, we briefly describe how controllers can be represented as decision trees as in 4]. We give an exemplified overview of the method, pinpointing the role of our algorithmic contributions.

A (non-deterministic, also called permissive) controller is a map $C: S \mapsto 2^{A}$ from states to non-empty sets of actions. This notion of a controller is fairly 
general; the only requirement is that it has to be memoryless and non-randomized. These kind of controllers are optimal for many tasks such as expected (discounted) reward, reachability or parity objectives. Moreover, even finite-memory controllers can be written in this form by considering the product of the state space with the finite memory as the domain, for example, like in LTL model checking.

Decision trees (DT), e.g. [38], are trees where every leaf node is labelled with a non-empty set of actions and every inner node is labelled with a predicate $\rho: S \mapsto\{$ true, false $\}$.

\begin{tabular}{rrrc}
\hline$v_{o}$ & $v_{f}$ & $\mathrm{~d}$ & actions \\
\hline 0 & 0 & 5 & $\{$ neu $\}$ \\
2 & 6 & 10 & $\{$ dec, neu, acc $\}$ \\
2 & 6 & 15 & $\{$ dec, neu, acc $\}$ \\
4 & 4 & 15 & $\{$ dec, neu $\}$ \\
\hline
\end{tabular}

(a)

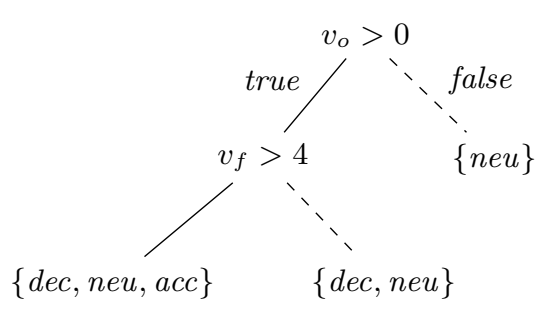

(b)

Fig. 1: An example controller based on the cruise-control model in the form of a lookup table (left), and the corresponding decision tree (right).

Example 2 (Decision tree representation). As an example, consider the controller given in Figure 1a. It is a subset of the real cruise-control case study from the motivating Example 1. A state is a 3-tuple of the variables $v_{o}, v_{f}$ and $d$, which denote the velocity of our car, the front car and the distance between the cars respectively. In each state, our car may be allowed to perform a subset of the following set of actions: decelerate (dec), stay in neutral (neu) or accelerate $(a c c)$. A DT representing this lookup table is depicted in Figure $1 \mathrm{~b}$

Given a state, for example $v_{o}=v_{f}=4, d=10$, the DT is evaluated as follows: We start at the root and, since it is an inner node, we evaluate its predicate $v_{o}>0$. As this is true, we follow the true branch and reach the inner node labelled with the predicate $v_{f}>4$. This is false, so we follow the false branch and reach the leaf node labelled $\{d e c, n e u\}$. Hence, we know that all three possibilities of decelerating, staying neutral and accelerating are allowed by the controller. $\triangle$

To construct a DT representation of a given controller, the following recursive algorithm may be used. Note that it is heuristic since constructing an optimal binary decision tree is an NP-complete problem [27.

Base case: If all states in the the controller agree on their set of actions $B$ (i.e. for all states $s$ we have $C(s)=B$ ), return a leaf node with label $B$.

Recursive case: Otherwise, we split the controller. For this, we select a predicate $\rho$ and construct an inner node with label $\rho$. Then we partition the controller 
by evaluating the predicate on the state space, and recursively construct one DT for the sub-controller on states $\{s \in S \mid \rho(s)\}$ where the predicate is true, and one for the sub-controller where it is false. These controllers are the children of the inner node with label $\rho$ and we proceed recursively.

For selecting the predicate, we consider two hyper-parameters: The domain of the predicates (see Section 45 and the way to select predicates (see Section 5). The selection is typically performed by selecting the predicate with the lowest impurity; this is a measure for how homogenous (or "pure") the controller is after the split, in other words the degree to which all the states agree on their actions.

We also consider a third hyper-parameter of the algorithm, namely determinization by safe early stopping (see Section 6). This modifies the base case as follows: if all states in the controller agree on at least one action $a$ (i.e. for all states $s$ we have $a \in C(s)$ ), then we return a leaf node with label $\{a\}$. This variant of early stopping ensures that, even though the controller is not represented exactly, still for every state a safe action is allowed.

Hence, if the original controller satisfies some property, e.g. that a safe set of states is never left, the DT construction algorithm ensures that this property is retained. This is because our algorithm represents the strategy exactly (or a safe subset, in case of determinization) and does not generalize as DTs typically do in machine learning. DTs are suitable for both tasks, as both rely on the strength of DTs exploiting underlying structure.

Remark 1. Note that for some types of objectives such as reachability, determinization of permissive strategies might lead to a violation of the original guarantees. For example, consider a strategy that allows both a self-looping and a non-self-looping action at a particular state. If the determinizer decides to restrict to the self-looping action, the reachability property may be violated in the determinized strategy. However, this problem can be addressed when synthesizing the strategy by ensuring that every action makes progress towards the target.

\section{Tool}

dtControl 2.0 is an easy-to-use open-source tool for representing memoryless symbolic controllers as more compact and more interpretable DTs, while retaining safety guarantees of the original controllers. Our website dtcontrol.model.in tum.de offers hyperlinks to the easy-to-install pip packag $£^{3}$ the documentation and the source code. Additionally, the artifact that has passed the TACAS 21 artifact evaluation is available here 6 .

The schema in Figure 2 illustrates the workflow of using dtControl, highlighting new features in red. Considering dtControl as a black box, it shows that given a controller, it returns a DT representing the controller and also offers the possibility to simulate a run of the system under the control of the DT, visualizing

${ }^{3}$ pip is a standard package-management system used to install and manage software packages written in Python. 


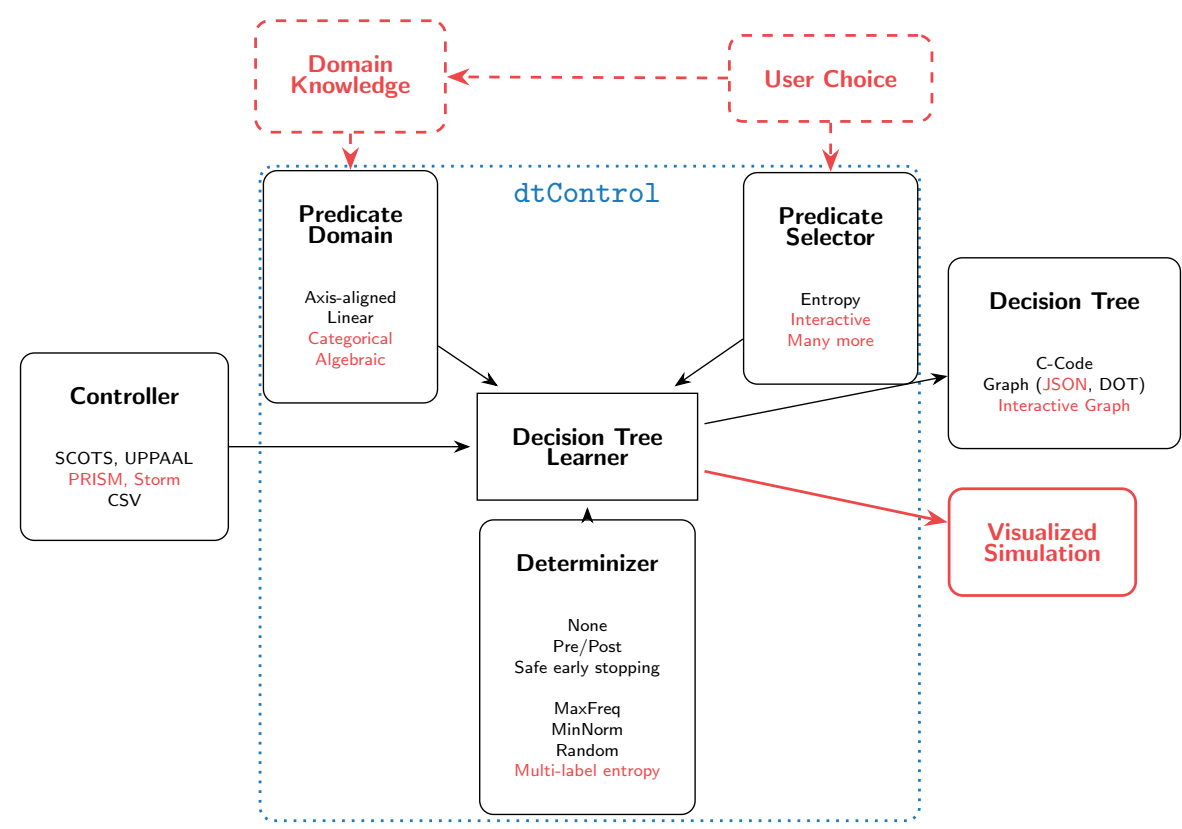

Fig. 2: An overview of the components of dtControl 2.0, thereby showing software architecture and workflow. Contributions of this paper are highlighted in red.

the decisions made. The controller can be input in various formats, including the newly supported strategy representations of the well-known probabilistic model checkers PRISM [32] and STORM [17]. The DT is output in several machine readable formats, and as C-code that can be directly used for executing the controller on embedded devices. Note that this C-code consists only of nested if-else-statements. The new graphical user interface also offers the possibility to inspect the graph in an interactive web user interface, which even allows to edit the DT. This means that parts of the DT can be retrained with a different set of hyper-parameters and directly replaced. This way, one can for example first train a determinized DT and then retrain important parts of it to be more permissive and hence more performant for a secondary criterion. Figure 3 shows a screenshot of the newly integrated graphical user interface.

Looking at the inner workings of dtControl, we see the three important hyper-parameters that were already introduced in Section 2 predicate domain, predicate selector, and determinizer. For each of these, dtControl offers various choices, some of which were newly added for version 2.0. Most prominently, the user now has the possibility to directly influence both the predicate domain and the predicate selector, by providing domain knowledge and thus also additional predicates, or by directly using the interactive predicate selection. More details on the predicate domain and how domain knowledge is specified can be found in Section 4. The different ways to select predicates, especially the new interactive mode, are the topic of Section 5 . Our new insights into determinization are 


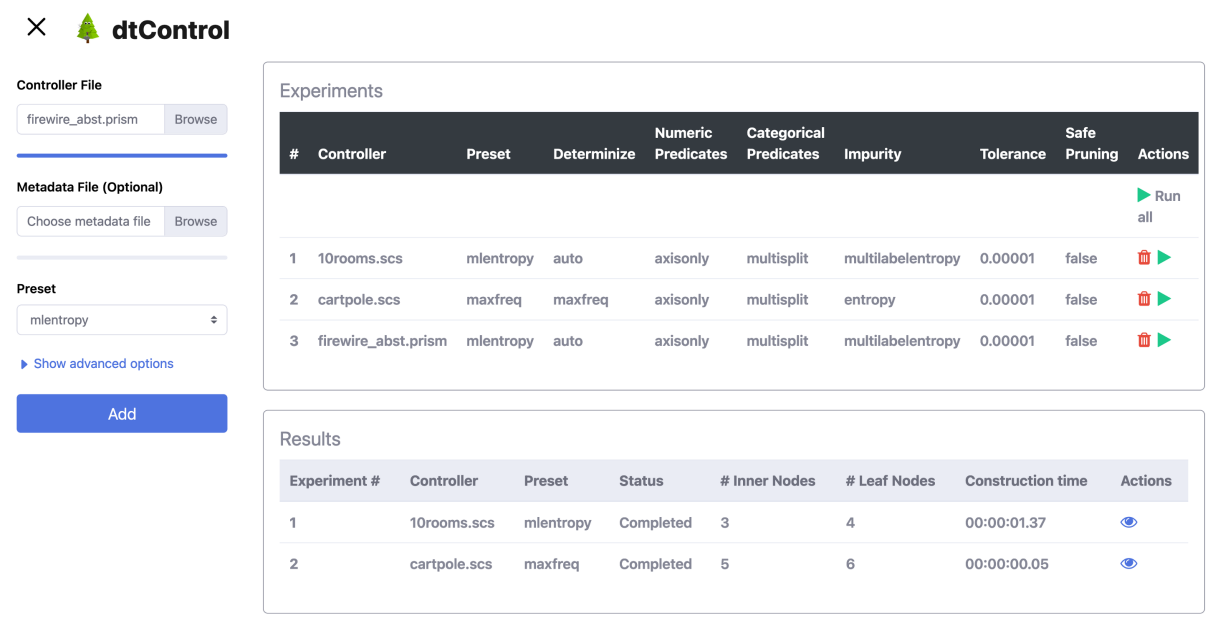

Fig. 3: Screenshot of the new web-based graphical user interface. It offers a sidebar for easy selection of the controller file and hyper-parameters, an experiments table where benchmarks can be queued, and a results table in which some statistics of the run are provided. Moreover, users can click on the 'eye' icon in the results table to inspect the built decision tree.

described in Section 6. To support the user in finding a good set of hyperparameters, dtControl also offers extensive benchmarking functionality, allowing to specify multiple variants and reporting several statistics.

Technical notes. dtControl 2.0 is written in Python 3 following an architecture closely resembling the schema in Figure 2 . The modularity, along with our technical documentation, allows users to easily extend the tool. For example, supporting another input format is only a matter of adding a parser.

dtControl 2.0 works with Python version 3.7.9 or higher. The core of the tool which runs the learning algorithms requires numpy [23], pandas [36] and scikit-learn 41] and optionally the library for the heuristic OC1 39. The algebraic predicates rely on SymPy [37] and SciPy [48. The web user interface is powered by Flask [1] and D3.js 9 .

\section{Predicate domain}

The domain of the predicates that we allow in the inner nodes of the DT is of key importance. As we saw in the motivating Example 1 allowing for more expressive predicates can dramatically reduce the size of the DT.

We assume that our state space is structured, i.e. it is a Cartesian product of the domain of the variables $\left(S=S_{1} \times \ldots \times S_{n}\right)$. We use $s_{i}$ to refer to the $i$-th state-variable of a state $s \in S$. In Example 2 the three state-variables are the velocity of our car, the velocity of the front car, and the distance. 
We first give an overview of the predicate domains dtControl 2.0 supports, before discussing the details of the new ones.

Axis-aligned predicates 38] have the form $s_{i} \leq c$, where $c$ is a rational constant. This is the easiest form of predicates, and they have the advantage that there are only finitely many, as the domain of every state-variable is bounded. However, they are also least expressive.

Linear predicates (also known as oblique [39]) have the form $\sum_{i} s_{i} \cdot a_{i} \leq c$, where $a_{i}$ are rational coefficients and $c$ is a rational constant. They have the advantage that they are able to combine several state-variables which can lead to saving linearly many splits, cf. 29 . Fig. 5.2]. The disadvantage of these predicates is that there are infinitely many choices of coefficients, which is why heuristics were introduced to determine a good set of predicates to try out 39,4 . However, heuristically determined coefficients and combinations of variables can impede explainability.

Algebraic predicates have the form $f(s) \leq c$, where $f$ is any mathematical function over the state-variables and $c$ is a rational constant. It can use elementary functions such as exponentiation, log, or even trigonometric functions. Example 1 illustrated how this can reduce the size and improve explainability. More discussion of these predicates follows in Section 4.2

Categorical predicates are special predicates for categorical (enumerationtype) state-variables such as colour or protocol state, and they are discussed in Section 4.1.

\subsection{Categorical predicates}

Categorical state-variables do not have a numeric domain, but instead are unordered and qualitative. They commonly occur in the models coming from the tools PRISM and STORM.

Example 3. Let one state-variable be 'colour' with the domain \{red, blue, green\}. A simple approach is to assign numbers to every value, e.g. red $=0$, blue $=$ 1 , green $=2$, and treat this variable as numeric. However, a resulting predicate such as colour $\leq 2$ is hardly explainable and additionally depends on the assignment of numbers. For example, it would not be possible to single out colour $\in\{$ red, green $\}$ using a single predicate, given the aforementioned numeric assignment. Using linear predicates, for example adding half of the colour to some other state-variable, is even more confusing and dependent on the numeric assignment.

Instead of treating the categorical variables using their numeric encodings, dtControl 2.0 supports specialized algorithms from literature, see e.g. 43, 44. They work by labelling an inner node with a categorical variable and performing a (possibly non-binary) split according to the value of the categorical variable. The node can have at most one child for every possible value of the categorical variable, but it can also group together similarly behaving values, see Figure 4 for an example. For the grouping, dtControl 2.0 uses the greedy algorithm from 44 . 
Chapter 7] called attribute-value grouping. It proceeds by first considering to have a branch for every single possible value of the categorical variable, and then merging branches as long as it improves the predicate; see [5. Appendix C] for the full pseudocode of the algorithm.

In our experiments we found that the grouping algorithm sometimes did not merge branches in cases where it would actually have made the DT smaller or more explainable. This is because the resulting impurity, the goodness of a predicate, could be marginally worse due to floating-point inaccuracies. Thus, we introduce tolerance, a bias parameter in favour of larger value groups. When checking whether to merge branches, we do not require the impurity to improve, but we allow it to become worse up to our tolerance. Setting tolerance to 0 corresponds exactly to the algorithm from [44], while setting tolerance to $\infty$ results in merging branches until only two remain, thus producing binary predicates.

To allow dtControl 2.0 to use categorical predicates, the user has to provide a metadata file, which tells the tool which variables are categorical and which are numeric; see 5, Appendix B.1] for an example.

\subsection{Algebraic predicates}

It is impossible to try out every mathematical expression over the state-variables, and it would also not necessarily result in an explainable DT. Instead, we allow the user to enter domain knowledge to suggest templates of predicates that dtControl 2.0 should try. See [5, Appendix B.2] for a discussion of the format in which domain knowledge can be entered.

Providing the basic equations that govern the model behaviour can already help in finding a good predicate, and is easy to do for a domain expert. Additionally, dtControl 2.0 offers several possibilities to further exploit the provided domain knowledge:

Firstly, the given predicates need not be exact, but may contain coefficients. These coefficients can be both completely arbitrary or may come from a finite set suggested by the user. For coefficients with finite domain, dtControl 2.0 tries all possibilities; for arbitrary coefficients, it uses curve fitting to find a good

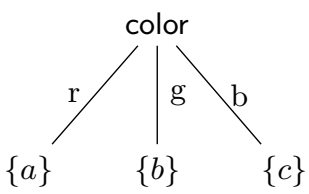

(a)

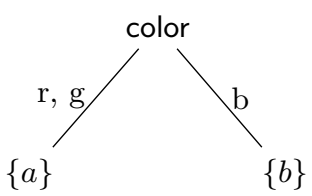

(b)

Fig. 4: Two examples of a categorical split. On the left, all possible values of the statevariable colour lead to a different child in a non-binary split. On the right, red and green lead to the same child, which is a result of grouping similar values together. 
value. For example, the user can specify a predicate such as $d+\left(v_{o}-v_{f}\right) \cdot c_{0}>c_{1}$ with $c_{0}$ being an arbitrary rational number and $c_{1} \in\{0,5,10\}$.

Secondly, the interactive predicate selection (see Section 5) allows the user to try out various predicates at once and observe their respective impurity in the current node. The user can then choose among them as well as iteratively suggest further predicates, inspired by those where the most promising results were observed.

Thirdly, the decisions given by a DT can be visualized in the simulator, possibly leading to better understanding the controller. Upon gaining any further insight, the user can directly edit any subtree of the result, possibly utilizing the interactive predicate selection again.

\section{Predicate selection}

The tool offers a range of options to affect the selection of the most appropriate predicate from a given domain.

Impurity measures: As mentioned in Section 2 the predicate selection is typically based on the lowest impurity induced. The most commonly used impurity measure (and the only one the first version of dtControl supported) is Shannon's entropy 46]. In dtControl 2.0, a number of other impurity measures from the literature [43, 13, 25, 39, 3] are available. However, our results indicate that entropy typically performs the best, and therefore it is used as the default option unless the user specifies otherwise. Due to lack of space, we delegate the details and experimental comparison between the impurity measures to [5, Appendix D].

Priorities: dtControl 2.0 also has the new functionality to assign priorities to the predicate generating algorithms. Priorities are rational numbers between 0 and 1 . The impurity of every predicate is divided by the priority of the algorithm that generated it. For example, a user can use axis-aligned splits with priority 1 and a linear heuristic with priority $1 / 2$. Then the more complicated linear predicate is only chosen if it is at least twice as good (in terms of impurity) as the easier-to-understand axis-aligned split. A predicate with priority 0 is only considered after all predicates with non-zero priority have failed to split the data. This allows the user to give just a few predicates from domain knowledge, which are then strictly preferred to the automatically generated ones, but which need not suffice to construct a complete DT for the controller.

Interactive predicate selection: dtControl 2.0 offers the user the possibility to manually select the predicate in every split. This way, the user can prefer predicates that are explainable over those that optimize the impurity.

The screenshot of the interactive interface in [5, Appendix F] shows the information that dtControl 2.0 provides. The user is given some statistics and metadata, e.g. minimum, maximum and step size of the state-variables in the current node, a few automatically generated predicates for reference and all 
predicates generated from domain knowledge. The user can specify new predicates and is immediately informed about their impurity. Upon selecting a predicate, the split is performed and the user continues in the next node.

The user can also first construct a DT using some automatic algorithm and then restart the construction from an arbitrary node using the interactive predicate selection to handcraft an optimized representation, or at any point decide that the rest of the DT should be constructed automatically.

\section{New insights about determinization}

In our context, determinization denotes a procedure that, for some or all states, picks a subset of the allowed actions. Formally, a determinization function $\delta$ transforms a controller $C$ into a "more determinized" $C^{\prime}$, such that for all states $s \in C$ we have $\emptyset \subsetneq C^{\prime}(s) \subseteq C(s)$. This reduces the permissiveness, but often also reduces the size. Note that, for safety controllers, this always preserves the original guarantees of the controller. For other (non-safety) controllers, see Remark 1.

dtControl 2.0 supports three different general approaches to determinizing a controller: pre-processing, post-processing and safe early stopping. Pre-processing commits to a single determinization before constructing the DT. Post-processing prunes the DT after its construction, e.g. safe pruning in [7]. The basic idea of safe early stopping is already described in Section 2. if all states agree on at least one action, then instead of continuing to split the controller, stop early and return a leaf node with that common action. Alternatively, to preserve more permissiveness, one can return not only a single common action, but all common actions; formally, return the maximum set $B$ such that for all states $s$ in the node $B \subseteq C(s)$.

The results of [4] show that both pre-processing and post-processing are outperformed by an on-the-fly approach based on safe early stopping. This is because pre-processing discards a lot of information that could have been useful in the DT construction and post-processing can only affect the bottom-most nodes of the resulting DT, but usually not those close to the root.

We now give a new view on safe early stopping approaches for determinizing a controller that allows us to generalize the techniques of [4], reducing the size of the resulting DTs even more.

Example 4. Consider the following controller: $C\left(s_{1}\right)=\{a, b, c\}, C\left(s_{2}\right)=\{a, b, d\}$, $C\left(s_{3}\right)=\{x, y\}$. All three states map to different sets of actions, and thus an impurity measure like entropy penalizes grouping $s_{1}$ and $s_{2}$ the same as grouping $s_{1}$ and $s_{3}$. However, if determinization is allowed, grouping $s_{1}$ and $s_{2}$ need not be penalized at all, as these states agree on some actions, namely $a$ and $b$. Grouping $s_{1}$ and $s_{2}$ into the same child node thus allows the algorithm to stop early at that point and return a leaf node with $\{a, b\}$, in contrast to grouping $s_{1}$ and $s_{3}$.

Knowing that we want to determinize by safe early stopping affects the predicate selection process. Intuitively, sets of states are more homogeneous the 
more actions they share. We want to take this into account when calculating the impurity of predicates. One way to do this would be to calculate the impurity of all possible determinization functions and pick the best one. This, however, is infeasible, hence we propose the heuristic of multi-label impurity measures. These impurity measures do not only consider the full set of allowed actions in their calculation, but instead they depend on the individual actions occurring in the set. This allows the DT construction to pick better predicates, namely those whose resulting children are more likely to be determinizable. In [5, Appendix E] we formally derive the multi-label variants of entropy and Gini-index.

To conclude this section, we point out the key difference between the new approach of multi-label impurity measures and the previous idea that was introduced in [4]. The approach from [4] does not evaluate the impurity of all possible determinization functions, but rather picks a smart one - that of maximum frequency (MaxFreq) - and evaluates according to that. MaxFreq determinizes in the following way: for every state, it selects from the allowed actions that action occurring most frequently throughout the whole controller. This way, many states share common actions. This is already better than pre-processing, as it does not determinize the controller a priori, but rather considers a different determinization function at every node. However, in every node we calculate the impurity for several different predicates, and the optimal choice of determinization function depends on the predicate. Thus, choosing a single determinization function for a whole node is still too coarse, as it is fixed independent of the considered predicate. We illustrate the arising problem in the following Example 5

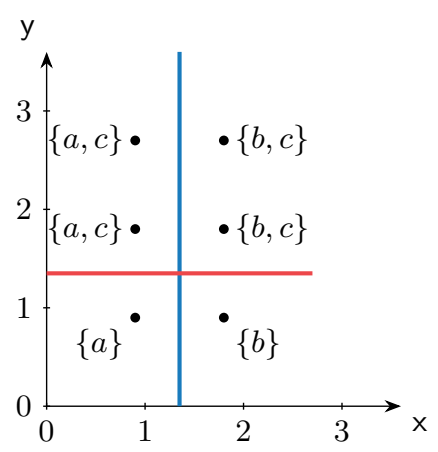

Fig. 5: A simple example of a dataset that is split suboptimally by the MaxFreq approach from 4], but optimally by the new multi-label entropy approach.

Example 5. Figure 5 shows a simple controller with a two-dimensional state space. Every point is labeled with its set of allowed actions.

As $c$ is the most frequent action, MaxFreq determinizes the states $(1,2)$, $(1,3),(2,2)$ and $(2,3)$ to action $c$. Hence the red split (predicate $y<1.5)$ is considered optimal, as it groups together all four states that map to $c$. The blue 
split (predicate $x<1.5$ ) is considered suboptimal, as then the data still looks very heterogeneous. So, using MaxFreq, we need two splits for this controller; one to split of all the $c$ 's and one to split the two remaining states.

However, it is better to first choose a predicate and then determine a fitting determinization function. When calculating the impurity of the blue split, we can choose to determinize all states with $x=1$ to $\{a\}$ and all states with $x=2$ to $\{b\}$. Thus, in both resulting sub-controllers the impurity is 0 as all states agree on at least one action. This way, one split suffices to get a complete DT. Multi-label impurity measures notice when labels are shared between many (or all) states in a sub-controller, and thus they allow to prefer the optimal blue split.

\section{Experiments}

Experimental setup. We compare three approaches: BDDs, the first version of dtControl from 4 and dtControl 2.0. For BDD 4 the variable ordering is important, so we report the smallest of 20 BDDs that we constructed by starting with a random initial variable ordering and reordering until convergence. To determinize BDDs, we used the pre-processing approach, 10 times with the minimum norm and 10 times with MaxFreq. For the previous version of dtControl, we picked the smaller of either a DT with only axis-aligned predicates or a DT with linear predicates using the logistic regression heuristic that was typically best in 4]. Determinization uses safe early stopping with the MaxFreq approach. For dtControl 2.0, we use the multi-label entropy based determinization and utilize the categorical predicates for the case studies from probabilistic model checking. We ran all experiments on a server with operating system Ubuntu 19.10, a 2.2GHz Intel(R) Xeon(R) CPU E5-2630 v4 and 250 GB RAM.

Comparing determinization techniques on cyber-physical systems. Table 1 shows the sizes of determinized BDDs and DTs on the permissive controllers of the tools SCOTS and Uppaal Stratego that were already used in 4]. We see that the new determinization approach is strictly better than the previous one, with only two DTs being of equal size, as the result of the previous method was already optimal. With the exception of the case studies helicopter and truck_trailer where BDDs are comparable or slightly better, both approaches using DTs are orders of magnitude smaller than BDDs or an explicit representation of the state-action mapping.

Case studies from probabilistic model checking. For Table 2 we used case studies from the quantitative verification benchmark set [24], which includes models from the PRISM benchmark suite 33]. Note that these case studies contain unordered enumeration-type state-variables for which we utilize the new categorical predicates. To get the controllers, we solved the case study with STORM and exported the resulting controller. This export already eliminates unreachable states. The

${ }^{4}$ Our implementation of BDDs is based on the dd python library https://github com/tulip-control/dd. 
Table 1: Controller sizes of different determinized representations of the controllers from SCOTS and Uppaal Stratego. "States" is the number of states in the controller, "BDD" the number of nodes of the smallest BDD from 20 tries, dtControl 1.0 [4] the smallest DT the previous version of dtControl could generate and dtControl 2.0 the smallest DT the new version can construct. "TO" denotes a failure to produce a result in 3 hours. The smallest numbers in each row are highlighted.

\begin{tabular}{|c|c|c|c|c|}
\hline Case study & States & BDD & dtControl 1.0 & dtControl 2.0 \\
\hline cartpole & 271 & 127 & 11 & 7 \\
\hline 10rooms & 26,244 & 128 & 7 & 7 \\
\hline helicopter & 280,539 & 870 & 221 & 123 \\
\hline cruise-latest & 295,615 & 1,448 & 3 & 3 \\
\hline dcdc & 593,089 & 381 & 9 & 5 \\
\hline truck_trailer & $1,386,211$ & 18,186 & 42,561 & 31,499 \\
\hline traffic_30m & $16,639,662$ & TO & 127 & 97 \\
\hline
\end{tabular}

previous version of dtControl was not able to handle these case studies, so we only compare dtControl 2.0 to BDDs.

Table 2 shows that also for case studies from probabilistic model checking, DTs are a good way of representing controllers. The DT is the smallest representation on 13 out of 19 case studies, often reducing the size by an order of magnitude compared to BDDs or the explicit representation. On 3 case studies, BDDs are smallest, and on 2 case studies, both the DT and the BDD fail to reduce the size compared to the explicit representation. This happens if there are many different actions and thus states cannot be grouped together. A worst case example of this is a model where every state has a different action; then, a DT would have as many leaf nodes as there are states, and hence twice as many nodes in total.

Remark 2. Note that the controllers exported by STORM are deterministic, so no determinization approach can be utilized in the DT construction. We conjecture that if a permissive strategy was exported, dtControl 2.0 would benefit from the additional information and be able to reduce the controller size further as for the cyber-physical systems.

\section{Conclusion}

We have presented a radically new version of the tool dtControl for representing controllers by decision trees. The tool now features a graphical user interface, allowing both experts and non-experts to conveniently interact with the decision tree learning process as well as the resulting tree. There is now a range of possibilities on how the user can provide additional information. The algebraic predicates provide the means to capture the (often non-linear) relationships from the domain knowledge. The categorical predicates together with the interface to probabilistic model checkers allow for efficient representation of strategies for Markov decision processes, too. Finally, the more efficient determinization yields 
Table 2: Controller sizes of different representations of controllers from the quantitative verification benchmark set 24], i.e. from the tools STORM and PRISM. "States" is the number of states in the controller, "BDD" the number of nodes of the smallest BDD of 20 tries and dtControl 2.0 the smallest DT we could construct. The smallest numbers in each row are highlighted.

\begin{tabular}{|c|c|c|c|}
\hline Case study & States & $\mathrm{BDD}$ & dtControl 2.0 \\
\hline triangle-tireworld.9 & 48 & 51 & 23 \\
\hline pacman. 5 & 232 & 330 & 33 \\
\hline rectangle-tireworld.11 & 241 & 498 & 373 \\
\hline philosophers-mdp.3 & 344 & 295 & 181 \\
\hline firewire_abst.3.rounds & 610 & 61 & 25 \\
\hline rabin. 3 & 704 & 303 & 27 \\
\hline ij.10 & 1,013 & 436 & 753 \\
\hline zeroconf.1000.4.true.correct_max & 1,068 & 386 & 63 \\
\hline blocksworld.5 & 1,124 & 3,985 & 855 \\
\hline cdrive. 10 & 1,921 & 5,134 & 2,401 \\
\hline consensus.2.disagree & 2,064 & 138 & 67 \\
\hline beb.3-4.LineSeized & 4,173 & 913 & 59 \\
\hline csma.2-4.some_before & 7,472 & 1,059 & 103 \\
\hline eajs.2.100.5.ExpUtil & 12,627 & 1,315 & 153 \\
\hline elevators.a-11-9 & 14,742 & 6,750 & 9,883 \\
\hline exploding-blocksworld.5 & 76,741 & 34,447 & 1,777 \\
\hline echoring.MaxOffline1 & 104,892 & 43,165 & 1,543 \\
\hline wlan_dl.0.80.deadline & 189,641 & 5,738 & 2,563 \\
\hline pnueli-zuck.5 & 303,427 & 50,128 & 150,341 \\
\hline
\end{tabular}

very small (possibly non-performant) controllers, which are particularly useful for debugging the model.

We see at least two major promising future directions. Firstly, synthesis of predicates could be made more automatic using mathematical reasoning on the domain knowledge, such as substituting expressions with a certain unit of measurement into other domain equations in the places with the same unit of measurement, e.g. to plug difference of two velocities into an equation for velocity. Secondly, one could transform the controllers into possibly entirely different controllers (not just less permissive) so that they still preserve optimality (or yield $\varepsilon$-optimality) but are smaller or simpler. Here, a closer interaction loop with the model checkers might lead to efficient heuristics. 


\section{References}

1. Flask web development: developing web applications with python. https://pypi org/project/Flask/, accessed: 14.10.2020

2. Adadi, A., Berrada, M.: Peeking inside the black-box: A survey on explainable artificial intelligence (XAI). IEEE Access 6, 52138-52160 (2018)

3. Ashok, P., Brázdil, T., Chatterjee, K., Křetínský, J., Lampert, C.H., Toman, V.: Strategy representation by decision trees with linear classifiers. In: QEST. Lecture Notes in Computer Science, vol. 11785, pp. 109-128. Springer (2019)

4. Ashok, P., Jackermeier, M., Jagtap, P., Křetínský, J., Weininger, M., Zamani, M.: dtcontrol: decision tree learning algorithms for controller representation. In: HSCC. pp. 17:1-17:7. ACM (2020)

5. Ashok, P., Jackermeier, M., Křetínský, J., Weinhuber, C., Weininger, M., Yadav, M.: dtControl 2.0: Explainable strategy representation via decision tree learning steered by experts. CoRR abs/2101.07202 (2021)

6. Ashok, P., Jackermeier, M., Křetínský, J., Weinhuber, C., Weininger, M., Yadav, M.: dtControl 2.0: Explainable strategy representation via decision tree learning steered by experts (TACAS 21 artifact) (Jan 2021). https://doi.org/10.5281/zenodo.4437169

7. Ashok, P., Křetínský, J., Larsen, K.G., Coënt, A.L., Taankvist, J.H., Weininger, M.: SOS: safe, optimal and small strategies for hybrid Markov decision processes. In: QEST. Lecture Notes in Computer Science, vol. 11785, pp. 147-164. Springer (2019)

8. Bahar, R.I., Frohm, E.A., Gaona, C.M., Hachtel, G.D., Macii, E., Pardo, A., Somenzi, F.: Algebraic decision diagrams and their applications. Formal Methods Syst. Des. 10(2/3), 171-206 (1997)

9. Bostock, M., Ogievetsky, V., Heer, J.: $\mathrm{D}^{3}$ data-driven documents. IEEE transactions on visualization and computer graphics 17(12), 2301-2309 (2011)

10. Boutilier, C., Dearden, R., Goldszmidt, M.: Exploiting structure in policy construction. In: IJCAI. pp. 1104-1113. Morgan Kaufmann (1995)

11. Brázdil, T., Chatterjee, K., Chmelik, M., Fellner, A., Křetínský, J.: Counterexample explanation by learning small strategies in Markov decision processes. In: CAV (1). Lecture Notes in Computer Science, vol. 9206, pp. 158-177. Springer (2015)

12. Brázdil, T., Chatterjee, K., Křetínský, J., Toman, V.: Strategy representation by decision trees in reactive synthesis. In: TACAS (1). Lecture Notes in Computer Science, vol. 10805, pp. 385-407. Springer (2018)

13. Breiman, L., Friedman, J.H., Olshen, R.A., Stone, C.J.: Classification and Regression Trees. Wadsworth (1984)

14. Bryant, R.E.: Graph-based algorithms for boolean function manipulation. IEEE Trans. Computers 35(8), 677-691 (1986)

15. Cimatti, A., Roveri, M., Traverso, P.: Automatic obdd-based generation of universal plans in non-deterministic domains. In: AAAI/IAAI. pp. 875-881. AAAI Press / The MIT Press (1998)

16. David, A., Jensen, P.G., Larsen, K.G., Mikucionis, M., Taankvist, J.H.: Uppaal stratego. In: TACAS. Lecture Notes in Computer Science, vol. 9035, pp. 206-211. Springer (2015)

17. Dehnert, C., Junges, S., Katoen, J., Volk, M.: A storm is coming: A modern probabilistic model checker. In: CAV (2). Lecture Notes in Computer Science, vol. 10427, pp. 592-600. Springer (2017)

18. Della Penna, G., Intrigila, B., Lauri, N., Magazzeni, D.: Fast and compact encoding of numerical controllers using obdds. In: Cetto, J.A., Ferrier, J.L., Filipe, J. (eds.) 
Informatics in Control, Automation and Robotics: Selcted Papers from the International Conference on Informatics in Control, Automation and Robotics 2008, pp. 75-87. Springer Berlin Heidelberg, Berlin, Heidelberg (2009)

19. Frehse, G., Guernic, C.L., Donzé, A., Cotton, S., Ray, R., Lebeltel, O., Ripado, R., Girard, A., Dang, T., Maler, O.: Spaceex: Scalable verification of hybrid systems. In: CAV. Lecture Notes in Computer Science, vol. 6806, pp. 379-395. Springer (2011)

20. Fujita, M., McGeer, P.C., Yang, J.C.: Multi-terminal binary decision diagrams: An efficient data structure for matrix representation. Formal Methods Syst. Des. 10(2/3), 149-169 (1997)

21. Garg, P., Neider, D., Madhusudan, P., Roth, D.: Learning invariants using decision trees and implication counterexamples. In: POPL. pp. 499-512. ACM (2016)

22. Girard, A.: Low-complexity quantized switching controllers using approximate bisimulation. CoRR abs/1209.4576 (2012)

23. Harris, C.R., Millman, K.J., van der Walt, S., Gommers, R., Virtanen, P., Cournapeau, D., Wieser, E., Taylor, J., Berg, S., Smith, N.J., Kern, R., Picus, M., Hoyer, S., van Kerkwijk, M.H., Brett, M., Haldane, A., del Río, J.F., Wiebe, M., Peterson, P., Gérard-Marchant, P., Sheppard, K., Reddy, T., Weckesser, W., Abbasi, H., Gohlke, C., Oliphant, T.E.: Array programming with numpy. CoRR abs/2006.10256 (2020)

24. Hartmanns, A., Klauck, M., Parker, D., Quatmann, T., Ruijters, E.: The quantitative verification benchmark set. In: TACAS (1). Lecture Notes in Computer Science, vol. 11427, pp. 344-350. Springer (2019)

25. Heath, D.G., Kasif, S., Salzberg, S.: Induction of oblique decision trees. In: Proceedings of the 13th International Joint Conference on Artificial Intelligence. Chambéry, France, August 28 - September 3, 1993. pp. 1002-1007 (1993)

26. Hoey, J., St-Aubin, R., Hu, A.J., Boutilier, C.: SPUDD: stochastic planning using decision diagrams. In: UAI. pp. 279-288. Morgan Kaufmann (1999)

27. Hyafil, L., Rivest, R.L.: Constructing optimal binary decision trees is NP-complete. Inf. Process. Lett. 5(1), 15-17 (1976)

28. Ittner, A., Schlosser, M.: Non-linear decision trees - NDT. In: ICML. pp. 252-257. Morgan Kaufmann (1996)

29. Jackermeier, M.: dtControl: Decision Tree Learning for Explainable Controller Representation. Bachelor's thesis, Technische Universität München (2020)

30. Jr., M.M., Davitian, A., Tabuada, P.: PESSOA: A tool for embedded controller synthesis. In: CAV. Lecture Notes in Computer Science, vol. 6174, pp. 566-569. Springer (2010)

31. Kiesbye, J.: Private Communication (2020)

32. Kwiatkowska, M.Z., Norman, G., Parker, D.: PRISM 4.0: Verification of probabilistic real-time systems. In: CAV. Lecture Notes in Computer Science, vol. 6806, pp. 585-591. Springer (2011)

33. Kwiatkowska, M.Z., Norman, G., Parker, D.: The PRISM benchmark suite. In: QEST. pp. 203-204. IEEE Computer Society (2012)

34. Larsen, K.G., Mikucionis, M., Taankvist, J.H.: Safe and optimal adaptive cruise control. In: Correct System Design. Lecture Notes in Computer Science, vol. 9360, pp. 260-277. Springer (2015)

35. Luttenberger, M., Meyer, P.J., Sickert, S.: Practical synthesis of reactive systems from LTL specifications via parity games. Acta Informatica 57(1-2), 3-36 (2020)

36. Wes McKinney: Data Structures for Statistical Computing in Python. In: Stéfan van der Walt, Jarrod Millman (eds.) Proceedings of the 9th Python in Science Conference. pp. 56 - 61 (2010). https://doi.org/10.25080/Majora-92bf1922-00a 
37. Meurer, A., Smith, C.P., Paprocki, M., Certík, O., Kirpichev, S.B., Rocklin, M., Kumar, A., Ivanov, S., Moore, J.K., Singh, S., Rathnayake, T., Vig, S., Granger, B.E., Muller, R.P., Bonazzi, F., Gupta, H., Vats, S., Johansson, F., Pedregosa, F., Curry, M.J., Terrel, A.R., Roucka, S., Saboo, A., Fernando, I., Kulal, S., Cimrman, R., Scopatz, A.M.: Sympy: symbolic computing in python. PeerJ Comput. Sci. 3, e103 (2017)

38. Mitchell, T.M.: Machine learning. McGraw Hill series in computer science, McGrawHill (1997)

39. Murthy, S.K., Kasif, S., Salzberg, S., Beigel, R.: OC1: A randomized induction of oblique decision trees. In: AAAI. pp. 322-327. AAAI Press / The MIT Press (1993)

40. Neider, D., Markgraf, O.: Learning-based synthesis of safety controllers. In: FMCAD. pp. 120-128. IEEE (2019)

41. Pedregosa, F., Varoquaux, G., Gramfort, A., Michel, V., Thirion, B., Grisel, O., Blondel, M., Prettenhofer, P., Weiss, R., Dubourg, V., Vanderplas, J., Passos, A., Cournapeau, D., Brucher, M., Perrot, M., Duchesnay, E.: Scikit-learn: Machine learning in Python. Journal of Machine Learning Research 12, 2825-2830 (2011)

42. Pyeatt, L.D., Howe, A.E., et al.: Decision tree function approximation in reinforcement learning. In: Proceedings of the third international symposium on adaptive systems: evolutionary computation and probabilistic graphical models. vol. 2, pp. 70-77. Cuba (2001)

43. Quinlan, J.R.: Induction of decision trees. Mach. Learn. 1(1), 81-106 (1986)

44. Quinlan, J.R.: C4.5: Programs for Machine Learning. Morgan Kaufmann (1993)

45. Rungger, M., Zamani, M.: SCOTS: A tool for the synthesis of symbolic controllers. In: HSCC. pp. 99-104. ACM (2016)

46. Shannon, C.E.: A mathematical theory of communication. Bell Syst. Tech. J. 27(4), 623-656 (1948)

47. St-Aubin, R., Hoey, J., Boutilier, C.: APRICODD: approximate policy construction using decision diagrams. In: NIPS. pp. 1089-1095. MIT Press (2000)

48. Virtanen, P., Gommers, R., Oliphant, T.E., Haberland, M., Reddy, T., Cournapeau, D., Burovski, E., Peterson, P., Weckesser, W., Bright, J., van der Walt, S., Brett, M., Wilson, J., Millman, K.J., Mayorov, N., Nelson, A.R.J., Jones, E., Kern, R., Larson, E., Carey, C.J., Polat, I., Feng, Y., Moore, E.W., VanderPlas, J., Laxalde, D., Perktold, J., Cimrman, R., Henriksen, I., Quintero, E.A., Harris, C.R., Archibald, A.M., Ribeiro, A.H., Pedregosa, F., van Mulbregt, P., SciPy: Scipy 1.0-fundamental algorithms for scientific computing in python. CoRR abs/1907.10121 (2019)

49. Zapreev, I.S., Verdier, C., Jr., M.M.: Optimal symbolic controllers determinization for BDD storage. In: ADHS 2018. IFAC-PapersOnLine, vol. 51, pp. 1-6. Elsevier (2018). https://doi.org/10.1016/j.ifacol.2018.08.001 
Open Access This chapter is licensed under the terms of the Creative Commons Attribution 4.0 International License(https://creativecommons.org/licenses/by/4 0/), which permits use, sharing, adaptation, distribution and reproduction in any medium or format, as long as you give appropriate credit to the original author(s) and the source, provide a link to the Creative Commons license and indicate if changes were made.

The images or other third party material in this chapter are included in the chapter's Creative Commons license, unless indicated otherwise in a credit line to the material. If material is not included in the chapter's Creative Commons license and your intended use is not permitted by statutory regulation or exceeds the permitted use, you will need to obtain permission directly from the copyright holder. 